\title{
Improved clinical outcomes following additional aggressive local consolidative therapy to systemic therapy for oligometastasis from lung cancer: where do we stand between "reality" and "illusion"?
}

\author{
Heerim Nam ${ }^{1}$, Jae Myoung Noh ${ }^{2}$, Seung Gyu Park ${ }^{3}$, Yong Chan Ahn ${ }^{2}$ \\ ${ }^{1}$ Department of Radiation Oncology, Kangbuk Samsung Hospital, Sungkyunkwan University School of Medicine, Seoul, 03181, Korea; ${ }^{2}$ Department \\ of Radiation Oncology, Samsung Medical Center, Sungkyunkwan University School of Medicine, Seoul, 06351, Korea; ${ }^{3}$ Department of Radiation \\ Oncology, Keimyung University Dongsan Medical Center, Keimyung University School of Medicine, Daegu, 42601, Korea \\ Correspondence to: Yong Chan Ahn, MD, PhD. Department of Radiation Oncology, Samsung Medical Center, Sungkyunkwan University School of \\ Medicine, Seoul, 06351, Korea. Email: ahnyc@skku.edu; ycahn.ahn@samsung.com. \\ Provenance: This is an invited article commissioned by the Section Editor Xiaozheng Kang (Department of Thoracic Surgery, Beijing Cancer \\ Hospital, Peking University, Beijing, China). \\ Comment on: Gomez DR, Tang C, Zhang J, et al. Local Consolidative Therapy Vs. Maintenance Therapy or Observation for Patients With Oligometastatic \\ Non-Small-Cell Lung Cancer: Long-Term Results of a Multi-Institutional, Phase II, Randomized Study. J Clin Oncol 2019;37:1558-65.
}

Submitted Sep 04, 2019. Accepted for publication Oct 08, 2019.

doi: $10.21037 /$ jtd.2019.10.18

View this article at: http://dx.doi.org/10.21037/jtd.2019.10.18

The concept of "oligometastatic state" has long been proposed and recognized based on several important clinical data (1-3). We have witnessed a growing concern and interest, and there are many publications that reported the clinical benefits by applying aggressive local therapy, in addition to the so-called standard palliative management policy among the patients with oligometastatic disease. Recently, the results of three phase 2 prospective randomized clinical trials that tried to verify the impact of aggressive local therapy, when compared to the standard maintenance systemic therapy alone, have been published (4-7). Brief comparisons of these three trials are summarized in Tables 1,2 .

In 2016, Gomez et al. (4) reported the results of phase 2 randomized trial, for the first time, which compared standard maintenance therapy with or without local consolidative therapy (LCT) applied to both the primary and metastatic lesions in the lung cancer patients with synchronous oligometastases. LCT included high dose radiation therapy (RT) and surgery, or combination of these two modalities. Gomez et al. (5) recently updated their long-term clinical results, and successfully confirmed the significantly improved progression-free survival (PFS) and overall survival (OS) by the early incorporation of LCT. They also reported that both the initial LCT before progression and the delayed LCT after progression contributed to the improved OS as well. In 2018, Iyengar et al. (6) reported the results of phase 2 randomized trial, which compared standard maintenance therapy with or without stereotactic ablative radiotherapy (SABR) in almost the same patients' population as Gomez's trial. Two trials by Gomez et al. and Iyengar et al. have a few important factors in common: lung cancer patients with synchronous oligometastasis; phase 2 trial to see the impact of the additional aggressive local therapy (LCT or SABR); and earlier trial closure than planned based on the significantly different clinical outcomes achieved. Though the definition of oligometastasis and the local therapy modalities were not the same, both trials reported almost tripled PFS advantage by adding aggressive local therapy. Gomez et al. (5) hypothesized that LCT could reduce the tumor burden having resistance to the initial systemic therapy, could potentiate the effect of subsequent systemic therapy, and could slow the growth of micrometastatic disease. The theoretical rationale of the Iyengar et al.'s regimen was based on the Norton-Simon hypothesis (6): 
Table 1 Comparison of study designs of three phase 2 randomized trials that tested impact of adding aggressive local therapy to standard maintenance therapy in oligometastatic lung cancer patients

\begin{tabular}{|c|c|c|c|}
\hline & lyengar et al. (6) & Gomez et al. $(4,5)$ & Palma et al. (7) \\
\hline $\begin{array}{l}\text { Number of participating } \\
\text { institute(s) }\end{array}$ & Single (UT Southwestern) & 3 from US and Canada & 10 from 4 non-US countries \\
\hline Primary site & Lung cancer only & Lung cancer only & Various sites ( $18 \%$ form lung ca) \\
\hline Initial Tx & $\begin{array}{l}\text { SD/PR to } 4-6 \text { cycles of } 1 \text { st-line } \\
\text { platinum-based chemotherapy }\end{array}$ & $\begin{array}{l}\text { SD/PR to } 1 \text { st-line systemic } T x \text { for } \geq 3 \\
\text { months }\end{array}$ & $\begin{array}{l}\text { Controlled primary }>3 \text { months following } \\
\text { definite therapy for primary }\end{array}$ \\
\hline Study duration & 2014 Apr-2016 Jul & 2012 Nov-2016 Jan & 2012 Feb-2016 Aug \\
\hline $\begin{array}{l}\text { Study design [Pts } \\
\text { number] }\end{array}$ & Phase 2, 1:1 randomization & Phase 2, 1:1 randomization & Phase 2, 1:2 randomization \\
\hline Primary end point & Progression-free survival & Progression-free survival & Overall survival \\
\hline \multirow[t]{4}{*}{ Secondary end points } & Toxicity & Overall survival & Quality of life \\
\hline & Local/distant control & Toxicity & Progression-free survival \\
\hline & Failure patterns & Appearance of new lesions & Lesional control \\
\hline & Overall survival & & $\begin{array}{l}\text { Number of further cycles of } \\
\text { chemotherapy or systemic therapy }\end{array}$ \\
\hline Follow-up & 9.6 months & 38.8 months & 25-26 months \\
\hline
\end{tabular}

SD, stable disease; PR, partial response; MT, maintenance therapy; SBRT, stereotactic radiotherapy; LCT, local consolidative therapy; SOC, standard of care.

Table 2 Comparison of clinical outcomes of three phase 2 randomized trials that tested impact of adding aggressive local therapy to standard maintenance therapy in oligometastatic lung cancer patients

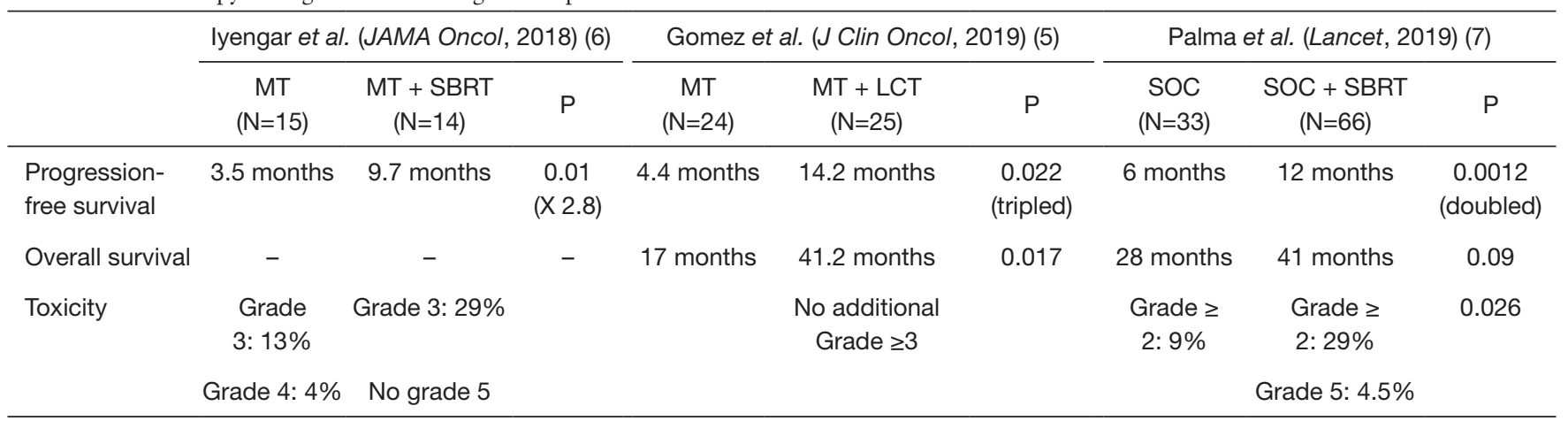

MT, maintenance therapy; SBRT, stereotactic radiotherapy; LCT, local consolidative therapy; SOC, standard of care.

the early consolidation with SABR, which is applied when the tumor burden is low but the growth rate is rapid, would be more advantageous than the delayed SABR after progression, when the tumor burden is high but the growth rate is slow.
Another recent publication by Palma et al. (7) deserves our attention in comparison to Gomez's and Iyengar's. Palma et al., by the phase 2 randomization trial, tried to see the impact of SBRT in addition to standard systemic therapy in 
the patients with oligometastases. Palma's trial is different from the above-mentioned 2 trials, in that they were focused only to the metachronous oligometastasis longer than 3 months with the controlled primary from various sites. Most commonly included primary sites were the breast, colorectal, lung and prostate, where the proportion of the lung cancer patients was $18 \%$. They reported doubling of PFS was by adding SBRT as a whole, and could have been biased by including the patients with primary sites, which would show less aggressive clinical behaviors than lung cancer. These three trials, so far, seem to be all currently available randomized clinical trials that affirmed the positive role of additional aggressive local therapy in treating the patients with oligometastasis from lung cancer.

Before commissioning to become aggressive, we had better answer a few important questions. First, how should we define "oligometastasis"? Iyengar and Palma defined the number of oligometastases as 5 or less, while Gomez accepted 3 or less. There is no perfect diagnostic tool in identifying the presence of very small metastatic foci as of yet, since every method naturally has false positive and false negative ratio. No one can propose a clear-cut, reasonable, and irresistible guideline on the number of metastatic lesions or the number of involved metastatic organs. It has been demonstrated that the longer the disease-free interval until oligometastasis, the clinical outcomes become better (8). Iyengar's and Gomez's trial from the beginning targeted the patients with synchronous oligometastasis, which means zero disease-free interval, and, however, were successful in achieving significantly improved PFS. Whether the improved PFS simply mean delaying progression needs to be further observed. Any proposal on the definition might be arbitrary. However, we need to reach a reasonable and acceptable consensus on the definition, methods of evaluation, and diagnostic criteria of "oligometastasis". Second, how high risk should we take? It is evident that the failure patterns have changed by the application of aggressive local therapy modality. We have witnessed significantly prolonged PFS and OS, which could have been achieved not only by the aggressive local therapy but also by more effective systemic therapy regimens than the past. Fortunately enough, trials that included only lung cancer patients reported no significantly added morbidity, while more frequent morbidities were observed in Palma et al.'s trial. We have to admit that doing nothing is often better than doing something having potential risk, and therefore, need to balance between the potential benefits and risks. The reasonable criteria on the selection of local therapy modality (RT or surgery) need to be developed. When RT is preferable, the guideline of RT detail (methods of target and organs-at-risk delineation, dose schedules, and techniques of RT, which usually vary depending on the site, tumor burden, histologic type, and patients' status) needs to be developed as well. In addition, when considering that the clinical outcomes should include not only the length of survival but also the quality of life, further investigation with this respect would be highly awaited. Third, how high cost incurred by the additional local therapy should we pay? The issue of "value" should always reside on the patients' side and should be based on the cost-effectiveness ratio, admitting that we do not have the unlimited resources allowed $(9,10)$. The optimal cost level needs to be determined not only on the basis of an individual but also on the society and system. Fourth, how the aggressive local therapy could be like when combined with immunotherapy? Though we have witnessed consistently improved clinical outcomes, all these trials may have been more or less outdated, when considering the recent paradigm shift towards the immunotherapy in lung cancer management. Collaboration of aggressive local therapy together with newer systemic therapy regimens, including immunotherapy, needs to be tested and verified in near future.

In brief, we need to accept the above-mentioned 3 important phase 2 trial results as a "reality" as of yet. And, at the same time, we need to be alert because this "reality" could be simply an "illusion". In this context, the results of the currently on-going large scale phase $2 / 3$ clinical trial (NRG-LU002) (11), which also will deal with the quality of life issue, is highly awaited.

\section{Acknowledgments}

None.

\section{Footnote}

Conflicts of Interest: The authors have no conflicts of interest to declare.

Ethical Statement: The authors are accountable for all aspects of the work in ensuring that questions related to the accuracy or integrity of any part of the work are appropriately investigated and resolved. 


\section{References}

1. Hellman S, Weichselbaum RR. Oligometastases. J Clin Oncol 1995;13:8-10.

2. Weichselbaum RR, Hellman S. Oligometastases revisited. Nat Rev Clin Oncol 2011;8:378-82.

3. Ashworth A, Rodrigues $\mathrm{G}$, Boldt $\mathrm{G}$, et al. Is there an oligometastatic state in non-small cell lung cancer? A systematic review of the literature. Lung Cancer 2013;82:197-203.

4. Gomez DR, Blumenschein GR Jr, Lee JJ, et al. Local consolidative therapy versus maintenance therapy or observation for patients with oligometastatic non-smallcell lung cancer without progression after first-line systemic therapy: a multicentre, randomised, controlled, phase 2 study. Lancet Oncol 2016;17:1672-82.

5. Gomez DR, Tang C, Zhang J, et al. Local Consolidative Therapy Vs. Maintenance Therapy or Observation for Patients With Oligometastatic Non-Small-Cell Lung Cancer: Long-Term Results of a Multi-Institutional, Phase II, Randomized Study. J Clin Oncol 2019;37:1558-65.

6. Iyengar P, Wardak Z, Gerber DE, et al. Consolidative Radiotherapy for Limited Metastatic Non-Small-Cell

Cite this article as: Nam H, Noh JM, Park SG, Ahn YC. Improved clinical outcomes following additional aggressive local consolidative therapy to systemic therapy for oligometastasis from lung cancer: where do we stand between "reality" and “illusion"? J Thorac Dis 2019;11(11):E217-E220. doi: 10.21037/ jtd.2019.10.18
Lung Cancer A Phase 2 Randomized Clinical Trial. JAMA Oncol 2018;4:e173501.

7. Palma DA, Olson R, Harrow S, et al. Stereotactic ablative radiotherapy versus standard of care palliative treatment in patients with oligometastatic cancers (SABRCOMET): a randomised, phase 2, open-label trial. Lancet 2019;393:2051-8.

8. Ashworth AB, Senan S, Palma DA, et al. An individual patient data metaanalysis of outcomes and prognostic factors after treatment of oligometastatic non-small-cell lung cancer. Clin Lung Cancer 2014;15:346-55.

9. Porter ME. What is value in health care? $\mathrm{N} \mathrm{Engl} \mathrm{J} \mathrm{Med}$ 2010;363:2477-81.

10. Rawlins MD. Cost, effectiveness, and value: how to judge? JAMA 2016;316:1447-8.

11. NRG-LU002. Maintenance Systemic Therapy Versus Local Consolidative Therapy (LCT) plus Maintenance Systemic Therapy for Limited Metastatic Non-Small Cell Lung Cancer (NSCLC): A Randomized Phase II/III Trial. In: ClinicalTrials.gov. Bethesda (MD): National Library of Medicine (US). NLM Identifier: NCT03137771. Available online: https://clinicaltrials. gov/ct2/show/NCT03137771 